\title{
Research on Design Method of Mechanical Systems Man-machine Interface
}

\author{
Fei Zhou ${ }^{1, a^{*}}$, Guomin Lin ${ }^{2, b}$, Miao Shang ${ }^{3, \mathrm{c}}$ and Changke Tan ${ }^{4, \mathrm{~d}}$ \\ College of Mechanical Engineering, Xijing University, Xi'an, China \\ a 743040357 @qq.com, b41922530@qq.com, \\ c445700839@qq.com, ${ }^{\mathrm{d}} 995965262 @ q q . c o m$
}

Keywords: Mechanical System; man-machine Interface; Optimal Degree of Matching; C++

\begin{abstract}
The traditional design method of man-machine interface of mechanical system is introduced, and the evaluation method of man-machine interface of mechanical system is put forward. On the basis of the evaluation of man-machine interface of mechanical system, the characteristics of man-machine interface are analyzed, and the optimization design method of man-machine interface of mechanical system is proposed. In the optimization design method, the single target function is geometric parameters of man-machine interface and geometric position of each element in the entire man-machine interface. The decorate area of Geometric location is as constraint conditions, the optimization method is combined with the stratified multi-objective optimization method and algorithm, in order to realize the system optimization of the man-machine interface.
\end{abstract}

\section{Introduction : Description of Man-machine Interface of Mechanical System}

Mechanical system of the man-machine interface (Man - Machine Interface) means the interaction between the operator and Machine area is the medium of transfer between computer and information [1]. It mainly includes three parts: Information on the display and man channel interface; Machine operator and organs in the interface of motion. People accept the outside information, material and energy through the sensory organs, and send message to the world, the material and energy through the executive organs of people. It can be thought that the machine and environment participating in all areas of the two processes belong to the man-machine interface.

\section{Traditional Design Method of Man-machine Interface of Mechanical System}

(1) According to the previous theories, research results and data, this paper analyzes the design of man-machine interface characteristics and its requirements, and follows the design personnel design experience and the analysis results of the man-machine interface design [2]. After the completion of the design, the researchers manufacture a prototype and test it. If the test results meet the design requirements, the test will be stopped. Otherwise, the design needs to be performed. It is the first time to use the design of the mechanical system of man-machine interface experiment method, this method is adapted to the simple man-machine interface design, and its disadvantage is that the design cycle is too long and wastes money severely.

(2) With the high-speed development of computer hardware and software, the man-machine interface designers began to apply the theory and method of artificial intelligence and expert system through the software programming as well as the ergonomics of the man-machine interface design idea, integrate theory and principles into the software of mechanical system on the man-machine interface design. Manufacturing prototype can be processed on computer design man-machine interface for modification, improvement, and trial production. However, the subjective and objective factors of man-machine interface design method are not considered comprehensively, Man-machine and man-machine interface participation is not evaluated, so there are still a lot of limitations. 


\section{Evaluation Method of Man-machine Interface of Mechanical System}

\section{Evaluation index}

The optimal degree of matching (ODOM) is an evaluation index. ODOM is made up of subjective evaluation and objective evaluation, including the general area, best concepts such as area in the ergonomics. At the same time, considering the importance element in the evaluation of human-computer interface and use objective factors, such as frequency, as well as the standard of the human body data, and so on [3]. Combined with means of weights, this paper uses the fuzzy comprehensive evaluation method for index calculation.

\section{Software Evaluation Method}

On the basis of ODOM, this paper uses a set of man-machine interface evaluation software system for mechanical systems HMIE (Human - Machine Interface Evaluation), which will be the programming language HMIE transplantation developed by $\mathrm{BC}++$ environment transferred to $\mathrm{VC}++$ development environment, and applies the optimization design to man-machine interface evaluation module into mechanical systems optimization design software system.

\section{Evaluation Method of Test}

The test evaluation method of ODOM is used for the man-machine interface on mechanical system through the test of man-machine interface of mechanical system [4], so as to seek an optimum matching scheme. Specific test evaluation method is as follows:

(1) Choose the right subjects

In order to increase the accuracy of the test evaluation and improve the accuracy of the test data, the researchers choose ergonomics expert as a subject in the test.

(2) Choose the appropriate test conditions

The researchers choose several typical working conditions of test conditions for experiments, and the participants determine the final interface matching.

(3) Match test

Under the guidance of the experimenter and instructions, the subjects are operating for the current test conditions in the test. After the completion of the operation, the participants get the comprehensive evaluation score for current system according to their own true feelings.

By means of the man-machine interface of mechanical system, the subjective evaluation results of test evaluation method on the man-machine interface can be obtained. Test evaluation method is a complement to the software evaluation method and it has been validated.

\section{Optimization Design Method of Man-machine Interface of Mechanical System}

\section{Optimization Design Theory}

Alleged optimization design, it is under the condition of certain constraints, to choose the appropriate design variables, to achieve the optimal value in according to the objective function [5]. There are two main elements for the optimization design: the first is that the actual technical problems become an optimization mathematical model, and the optimization design has completed the correct modeling.

For the optimization design mathematical model, the commonly used optimization algorithms include linear programming model, nonlinear programming model and quadratic programming model, the geometric programming model, multi-objective programming model, the dynamic programming model, etc.

\section{Optimization Design Problem Analysis of Man-machine Interface of Mechanical System.}

The variables of optimization design:

In the Man-machine or the discrete variables, it is generally assumed that they are continuous when finding the optimal value, and then get into integer or near a few selected according to the standard. When dealing with the problem, the researchers also use the integer programming.

The objective function of optimization design: 
The optimization design of the man-machine interface for mechanical system is based on man-machine interface evaluation [6]. Therefore, the mechanical overall evaluation index system of man-machine interface - system ODOM is an objective function, and each element ODOM of man-machine interface is a single objective function. ODOM is expressed as:

$$
(E s)_{i}=\frac{E s_{i}}{\sum_{i=1}^{n} E s_{i}}
$$

The expression is the objective function to optimize the design of man-machine interface.

\section{Optimization Design Constraints}

The constraint is to give some limitations of mathematical relationship. All the area of constraints can be satisfied, which is called the feasible region. Within the feasible region, all the constraint conditions are satisfied. Outside the feasible region, some constraints cannot be satisfied.

\section{Optimization Design Algorithm}

By using the optimization methods, the researchers can get the optimal solution of objective function. Due to the nature of the objective function, the constraint conditions are different [7]. According to the ideas of the mathematical programming, optimization problem can be divided into several categories: linear programming [8], nonlinear programming, geometric programming, dynamic programming, goal programming and multi-objective programming, integer programming, and so on.

The basic structure of the optimization method:

Optimization method for the optimal solution by using iterative method, set $x *$ for the first $\mathrm{k}$ iteration point, ${ }^{d}$ Search direction for the $\mathrm{k}$ times, ${ }^{a_{k}}$ is the $\mathrm{k}$ step length factor, and $\mathrm{k}$ time's iteration is as follows:

$$
x_{k+1}=x_{k}+a_{k} d_{k}
$$

Different step length factor $x *$ and different search directions $d_{k}$ constitute the different optimization method, which can be seen by iterative format. Search direction is the drop direction of objective function $\mathrm{f}(\mathrm{x})$ in $x *$ point, ${ }^{d_{k}}$ content:

$$
f\left(x_{k}+a_{k} d_{k}\right)<f\left(x_{k}\right)
$$

The determination of optimal design algorithm:

In the mechanical system with optimum design, man-machine interface is implemented according to the following steps:

(1) The ODOM of each component for man-machine interface is the objective function [9]. According to design principles of man-machine interface, the researchers can get the constraint conditions of each element. To optimize the geometry size of each element and get the optimal solution, each element in ODOM is biggest state;

(2) The ODOM of system of man-machine interface is objective function. Predecessors' research results locate each component layout constraints, and the geometric layout position of each element is optimized design, in order to obtain each element of the optimum geometrical arrangement position [10].

Evaluation of the algorithm of optimization design:

(1) Reliability

Under the reasonable accuracy, reliability of the design optimization algorithm is the success rate of the optimization problem within a certain number of iterations and the computation time.

(2) Effectiveness

Effectiveness is an efficient algorithm to solve some problems. The measures of effectiveness can be generally considered from the algorithm aspect, which is used in the computation time and the number of function evaluation. 
(3) Difficulty Level of Preparation Work

The difficulty of the preparatory work is considered into the evaluation algorithm. This is usually referred to calculate function derivative and the calculation procedure and simplified, etc.

(4) Convergence

Each algorithm must have a convergence algorithm. Otherwise, the algorithm will not be able to set up in theory, and it is not used for problem solving.

\section{Conclusions}

The traditional design method of man-machine interface of mechanical system is introduced in the paper, and the evaluation method of man-machine interface of mechanical system is put forward. On the basis of the evaluation of man-machine interface of mechanical system, this paper analyzes the characteristics of man-machine interface, and proposes the optimization design method of man-machine interface of mechanical system. In the optimization design method, the single target function is geometric parameters of man-machine interface and geometric position of each element in the entire man-machine interface. The decorate area of Geometric location is used as constraint conditions, and the optimization method is combined with stratified multi-objective optimization method and algorithm, in order to realize the system optimization of the man-machine interface. This paper uses the optimized design method of man-machine interface for the mechanical system, in order to realize the optimal design of man-machine interface, improve the design quality of man-machine interface of mechanical system and the design efficiency, shorten the design period of the related design personnel, and reduce the design cost.

\section{References}

[1] J.H. Zhao, Man-Machine Engineering. Higher Education Press, Beijing, 2006.01.

[2] Yao Jun, Based on Design of Economics Knowledge Cognition and Application Study. 3 (2010)5-8.

[3] L.Zhang, W.P.XU, Man-Machine Interface Design. Beijing Cambridge University Press, Beijing, 2012.

[4] J.Xiu. Coal Mining Machinery for the Optimization Design of the Man-machine Engineering. Journal of Coal Technology. 32(2013) 19-20.

[5] W.Xi. Man-machine Interface Configuration and Application Technology. Mechanical Industry Publishing House, Beijing, 2010.

[6] L.Han. The Man-machine Interface Design of Mechanical System. Computer Programming Skills and Maintenance. 2(2010)27 - 30.

[7] S.Zhou. Man-machine Interface Design. Science Press, Beijing, 2007.

[8] W.Yang. The Application of Ergonomics in the Vibratory Roller Design [J]. (2011)124-125.

[9] Q.A.Chen, Software Interface Design [M]. Higher Education Press, Beijing, 2008.

[10] J.Q.Guan, J.Q.Wen. Mechanical and Electrical Hydraulic Control Technology. Tongji University Press, Shanghai, 2010. 